\title{
Tecnologia Assistiva MINDER: possibilidades de um computador de baixo custo na inclusão sociodigital de sujeitos não videntes
}

\author{
André L. A. Rezende ${ }^{1}$, Cayo P. S. de Jesus ${ }^{2}$, Amanda E. S. Nogueira ${ }^{3}$, Isis B. S. \\ Pereira $^{4}$, Aderaldo C. da S. Neto ${ }^{5}$, Gustavo J. da S. Costa ${ }^{6}$, Márcio V. S. Souza ${ }^{7}$ \\ 1,2,4,5,6,7Instituto Federal de Educação, Ciência e Tecnologia Baiano (IF Baiano - Campus \\ Catu). CEP: 48.110-000 - Catu - BA - Brasil \\ ${ }^{3}$ Instituto Federal de Educação, Ciência e Tecnologia Baiano (IF Baiano - Reitoria) \\ CEP: 41.720-052 - Salvador-BA - Brasil
\{andre.rezende, cayo.santana, amanda.nogueira\} @ifbaiano.edu.br, isisbeatrizsp2010@hotmail.com, aderaldo.catarino@gmail.com, gustavojesus 365@outlook.com, marushio.bic@gmail.com

\begin{abstract}
Technological advances have made computing an essential element in everyone's daily life, making it essential for digital and social inclusion. When it comes to people with some type of disability, it becomes essential in this process. In this context, this article addresses the project that intends to develop a low-cost personal computer, named MINDER. The methodology is based on the Applied Collaborative Methodology and on the PDCA and PDSII methods. Still in the development phase, the research has as preliminary results the production of hardware and software, based on the participants' proposals and awaits the final phases for completion.
\end{abstract}

Resumo. $O$ avanço tecnológico fez da informática um elemento essencial no cotidiano de todos, tornando-a imprescindivel para a inclusão digital e social. Em se tratando das pessoas com algum tipo de deficiência, torna-se indispensável neste processo. Neste contexto, este artigo aborda o projeto que pretende desenvolver um computador pessoal de baixo custo, nomeado MINDER. A metodologia fundamenta-se na Metodologia Colaborativa Aplicada e nos métodos PDCA e PDSII. Ainda em fase de desenvolvimento, a pesquisa tem como resultados preliminares a produção do hardware e software, com base nas proposições dos participantes e aguarda as fases finais para conclusão. 


\section{Introdução}

A Tecnologias Digitais da Informação e Comunicação (TDIC) apresentam inúmeras aplicabilidades, dentre elas podemos citar, a título de exemplificação, a automação de processos, comunicação remota, realização de cálculos complexos, reconhecimento de imagens, entretenimento, simulação de eventos climáticos entre outras possibilidades. A utilização das TDIC foi incorporada rapidamente ao cotidiano por facilitar processos, promover o encurtamento de distâncias e possibilitar estar, conhecer, aprender, fazer diferentes ações em tempos e espaços diversos, tornando-se um elemento praticamente indispensável na contemporaneidade, para sujeitos com demandas das mais variadas.

A TDIC, por suas características e aplicações, pode ser potencializada quando adentramos no universo das pessoas com algum tipo de limitação, em particular do deficiente visual (cegos e/ou baixa visão). A condição em questão, se acentua por as TDIC possibilitarem maior autonomia e promoverem o desenvolvimento efetivo de habilidades intelectuais.

É justamente, perante este cenário, que advogamos a importância do estimulo no desenvolvimento de projetos que articulem produtos e/ou serviços, objetivando minimizar e/ou superar as dificuldades enfrentadas no dia a dia dos sujeitos com algum tipo de deficiência visual, possibilitando assim, estruturar as condições que favoreçam o processo de inclusão digital.

Em consonância a argumentação anterior, temos relatos de estudantes com deficiência visual, parcial (baixa visão) ou total (cegos), de suas dificuldades de acesso a computadores pessoais que possuam recursos de acessibilidade, preponderantemente pela questão financeira das famílias, tornando inviável o acesso aos aparatos tecnológicos. Em face desse cenário é que ora pensamos essa pesquisa.

Neste contexto, surgiram os seguintes questionamentos: Como desenvolver uma tecnologia assistiva de baixo custo que possibilite a interação das pessoas com deficiência visual com os diversos conteúdos disponibilizados em formato digital? Como maximizar o acesso dos deficientes visuais às informações existentes na rede mundial de computadores?

$\mathrm{Na}$ busca por essas respostas, está em desenvolvimento um projeto de pesquisa que visa criar um computador pessoal de baixo custo para cegos, nomeado como MINDER, traduzido tecnicamente pela interação entre um computador de placa única (Single Board Computers), a exemplo do Raspberry $\mathrm{Pi}^{1}$ ou similar, um sistema operacional e um leitor de telas, ambos gratuitos e de código aberto.

A próxima seção deste artigo apresenta as bases teóricas que alicerçam este trabalho, como a deficiência visual, as Tecnologias Assistivas na inclusão sociodigital das pessoas com deficiência visual e a solução proposta por este trabalho. A seção 4 revela a metodologia utilizada no desenvolvimento do projeto e por fim os resultados preliminares.

\footnotetext{
${ }^{1}$ Disponível em: <https://www.raspberrypi.org/>. Acesso em 05/07/2021.
} 


\section{Bases Teóricas}

\subsection{A caracterização do deficência visual}

Existem diversas definições e consequentemente diversas classificações para deficiência visual, uma vez que esta pode ser congênita ou adquirida. Para a Organização Mundial da Saúde - OMS (do inglês, World Health Organization - WHO) a deficiência visual "ocorre quando uma doença ocular afeta o sistema visual e uma ou mais funções visuais" (OMS, 2021, p. 10). O Decreto $\mathrm{n}^{\mathrm{o}}$ 5.296, de 2 de dezembro de 2004 (BRASIL, 2004), estabelece e classifica a deficiência visual em: a) cegueira: ocorre quando a acuidade visual é igual ou menor que 0,05 no melhor olho, com a melhor correção óptica; b) baixa visão: a acuidade visual entre 0,3 e 0,05 no melhor olho, com a melhor correção óptica; os casos nos quais a somatória da medida do campo visual em ambos os olhos for igual ou menor que 60 graus; ou a ocorrência simultânea de quaisquer das condições anteriores.

Existem diversos tipos de baixa visão, dentre os quais destacamos (LAVORATO, 2018): redução da visão central; diminuição da visão periférica (visão tubular); perda da visão das cores; incapacidade ou perda da aptidão do olho para se ajustar à luz, contraste, ou brilho, dentre outros. Isso significa que a depender do tipo de baixa visão, há uma ou mais consequências para o deficiente visual. Dentre essas, destacam-se (LAVORATO, 2018, p. 41): “a) Percepção Turva: os contrastes são poucos perceptíveis, as distâncias são de difícil mensuração, existe uma má percepção do relevo, as cores são atenuadas; b) Visão Periférica: maior dificuldade para distinguir objetos, símbolos ou caracteres pequenos, distantes e em baixo contraste com o ambiente; c) Visão Tubular ou central: pode ter acuidade visual normal na região central da retina; a visão noturna é reduzida; apresenta limitação na orientação espacial e nas atividades de autonomia".

Uma outra definição para deficiência visual é apresentada pela Classificação Internacional de Funcionalidade, Incapacidade e Saúde - CIF (do inglês, International Classification of Functioning, Disability and Health - ICF), desenvolvida pela OMS, pertencente às suas classificações internacionais para aplicação em vários aspectos da saúde. A aplicação desse modelo propicia a elaboração de uma base científica para a compreensão e o estudo da saúde, dos estados relacionados com a saúde, dos resultados e dos determinantes.

Destarte, o mesmo passa a funcionar como um mecanismo investigativo, no qual é possível medir resultados, a qualidade de vida ou os fatores ambientais. Para tal, a CIF leva em consideração as adversidades enfrentadas pelo deficiênte visual na sua capacidade, ou seja, o que esse indivíduo consegue fazer, considerando o estado das funções e estruturas de seu corpo. Outro ponto avaliado é o desempenho, isto é, a interação do sujeito com o ambiente, quais são os fatores que melhoram ou pioram a execução de uma determinada atividade. Esse contexto é analisado pela CIF, o qual pode ter resultados completamente divergentes para cada pessoa, uma vez que leva-se em consideração as influências dos fatores ambientais e pessoais (WHO, 2018).

Estudos (BOURNE et al. 2017) demonstram uma estimativa de 36 milhões da população mundial é cega e 216,6 milhões de pessoas possuem visão subnormal. No 
Brasil, de acordo com o censo $^{2}$ realizado pelo Instituto Brasileiro de Geografia e Estatística (IBGE), existem 45.623.910 (23,9\%) milhões de pessoas que possuem algum nível de dificuldade em ao menos uma das seguintes habilidades investigadas: enxergar, ouvir, caminhar, subir degraus ou possuir deficiência mental/intelectual (IBGE, 2012). Contudo, houve uma releitura desses dados em 2018 pelo IBGE, o qual procurou adaptá-los a CIF, em tempo que atendesse a concepção da Convenção de Direitos da Pessoa com Deficiência, aprovada pela Organização das Nações Unidas (ONU) em 13 de dezembro de 2006, promulgado pelo Brasil em 25 de agosto de 2009, através do Decreto n. 6.949.

Desse modo, houve uma tentativa de se adequar os dados com intuito de atender a padronização adotada pelo grupo de Washington para Estatísticas sobre Pessoas com Deficiência (do inglês, Washington Group on Disability Statistics - WG). O WG tem como objetivo assegurar a harmonia entre as estatísticas de diferentes países ao tempo que promove a cooperação internacional no âmbito das estatísticas de pessoas com deficiência.

Assim, conforme a releitura dos dados do censo, o Brasil passou a ter 12.748.663 pessoas, ou 6,7\%, com as dificuldades apresentadas anteriormente, em um universo de 190.755 .048 recenseadas. O maior impacto da reclassificação, foi no percentual de pessoas com deficiência visual que conforme o novo critério é de $3,4 \%$. A mudança nos novos critérios utilizados foi a adoção de um novo formato para identificar a pessoa com deficiência no conjunto de questões que passou de "considerando pessoa com deficiência os indivíduos que responderam ter pelo menos alguma dificuldade em uma ou mais questões" para "considerando pessoa com deficiência os indivíduos que responderam ter pelo menos muita dificuldade em uma ou mais questões" (IBGE, 2018, grifo nosso).

\title{
2.2 As tecnologias assistivas no contexto do deficiente visual
}

A tecnologia tem avançado de forma veloz, principalmente, no sentido de criar meios para que pessoas que possuem determinadas limitações sejam integradas à sociedade de forma menos traumática. Pode-se representar esse avanço, através do termo "Tecnologias Assistivas/ Adaptativas", que pode ser traduzido, de forma simples, como qualquer ferramenta ou aparato tecnológico com a finalidade de potencializar as habilidades de pessoas que possuem limitações sensoriais ou físicas, promovendo, portanto, uma maior independência do indivíduo.

Essa ferramenta pode ser um software, hardware, um equipamento desenvolvido de forma caseira ou em série, com o intuito de melhorar a interface dessas interações. Estas ferramentas adaptativas:

\begin{abstract}
referem-se ao conjunto de recursos que, de alguma maneira, contribuem para proporcionar às $\mathrm{PNEEs}^{3}$ maior independência, qualidade de vida e inclusão na vida social através do suplemento, manutenção ou devolução de suas capacidades funcionais" (Hogetop e Santarosa, 2002, p. 24).
\end{abstract}

Segundo Santarosa (2000), os suportes tecnológicos apresentam uma nova realidade, "principalmente para as pessoas cujos padrões de aprendizagem não seguem

\footnotetext{
${ }^{2}$ A última atualização dos dados é de 2010, pois o IBGE somente realiza o Censo Demográfico a cada dez anos.

${ }^{3}$ Pessoas com Necessidades Educativas Especiais
} 
os quadros típicos de desenvolvimento. Os estudos mostram que pessoas limitadas por deficiências não são menos desenvolvidas, mas se desenvolvem de forma diferente". Por isso as Tecnologias assistivas são necessárias.

Para Gonzáles (2007, p. 111), sobre os não videntes, "as melhores áreas de intervenção incluem a prevenção, o desenvolvimento de habilidades, a aprendizagem para o uso de mobilidades alternativas, o treinamento em orientação e mobilidade". E complementa afirmando que, em se tratando de intervenção, há diferentes níveis de atuação: individual, coletiva, médica, técnica e educacional.

Com base nessas referências, podemos afirmar que o MINDER trata-se de um recurso de intervenção educacional pelo contexto de onde partiu a pesquisa, assim como pela intenção de produzir melhor qualidade no atendimento educacional e possibilitar maior permanência escolar aos não videntes, entretanto a intenção do MINDER é favorecer a inclusão digital, pois o uso das tecnologias pode minimizar as barreiras impostas por uma sociedade criada para a pessoa dita normal.

\subsection{MINDER: um computador pessoal de baixo custo como possibilidade de inclusão digital}

Existem alguns dispositivos direcionados em proporcionar computadores de baixo custo. O Raspberry Pi é um exemplo de um computador de placa única que foi concebido para ser o computador mais barato do mercado. O seu lançamento deu-se em 29 de fevereiro de 2012 com finalidades educacionais, em particular para estimular o ensino de programação e o uso da tecnologia. A adoção desta tecnologia no projeto MINDER deu-se em virtude das características a seguir definidas:
1.Preço;
2.Certificado pela Agência Nacional de Telecomunicações;
3.Conector de áudio garantindo a utilização do fone de ouvido;
4.Conectores USB garantindo conectar outros periféricos, como o teclado;
5.Processamento de tarefas similar a de um computador de uso pessoal;
6.Integração com programas de acessibilidade, como o leitor de telas;
7.Conectividade à Internet por meio de redes sem fio ou cabo;
8.Comunidade ativa e em constante evolução;
9.Conectividade por meio de bluetooth.

O MINDER se sustenta na arquitetura do Raspberry Pi. Como pode ser observado na Figura 1, o protótipo é composto por três componentes visualmente identificáveis: o elemento indicado pela letra A, é o computador de placa única Raspberry $P i$ (versão 4.0). Neste hardware encontra-se disponível a memória RAM de $8 \mathrm{~GB}$, memória de armazenamento de $32 \mathrm{~GB}$; $\mathrm{O}$ elemento indicado pela letra $\mathrm{B}$, é um teclado flexível (borracha) elegido por ser portátil; $\mathrm{O}$ elemento indicado pela letra $\mathrm{C}$ é o fone de ouvido, item primordial para o funcionamento do aparato, pois permite ao deficiente visual "ouvir" as interações com a solução. 


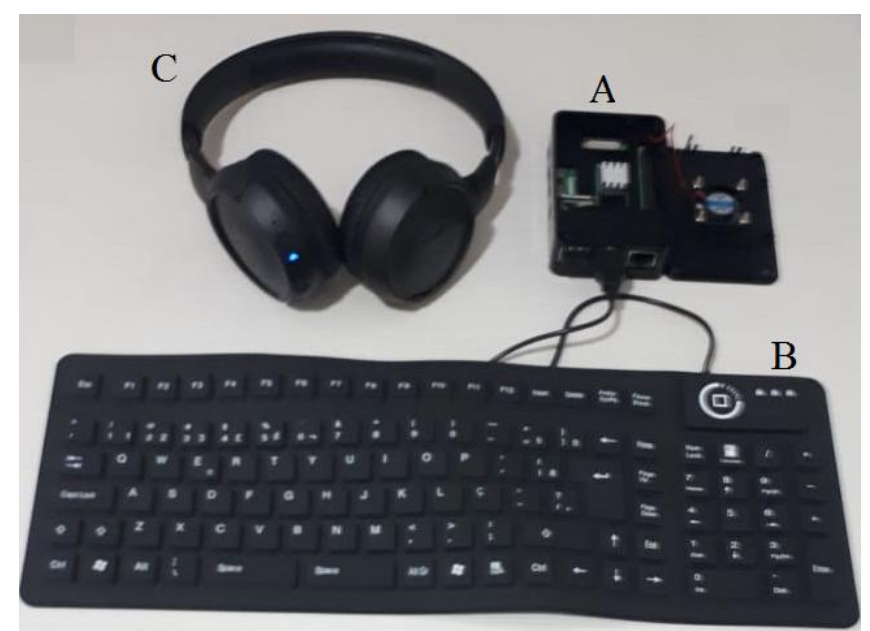

Figura 1. Protótipo do MINDER. Fonte: Autores

Em relação a definição dos softwares a serem instalados e configurados no MINDER, temos: o sistema operacional, um leitor de telas, um navegador e por fim, um pacote de ferramentas de escritório. Este conjunto de programas compõem a infraestrutura tecnológica mínima para que as pessoas com algum tipo de limitação visual possam operar e desenvolver as atividades inerentes ao dispositivo.

$\mathrm{Na}$ escolha do sistema operacional foram considerados os seguintes critérios:

1.Disponibilidade de tradução para o Português do Brasil;

2.Disponibilidade de interface gráfica;

3.Comunidade ativa;

4.Possibilidade de instalação de novos programas;

5.Melhor desempenho no teste de benchmark $^{4}$.

O Raspberry Pi OS é o sistema operacional oficial para todos os modelos do Raspberry Pi. No entanto, são indicadas outras distribuições na página da Raspberry Fundation, são eles: Ubuntu Mate, Ubuntu Core, Ubuntu Server, OSMC, LibreELEC, Mozilla Web Things, PiNet e RISC OS.

Dentre os sistemas operacionais citados anteriormente, apenas o Raspberry Pi OS e o Ubuntu Mate possuem a finalidade de serem utilizados em computadores pessoais. Ou seja, apresentam interface gráfica, acesso a conteúdos da Internet através de um navegador e possuem ferramentas de escritório, a exemplo do editor de planilha, texto e apresentação.

$\mathrm{Na}$ tentativa de ampliar o leque de possibilidades relacionadas aos sistemas operacionais disponíveis para computadores de uso pessoal, foram selecionadas algumas distribuições extra-oficiais, são elas: LinuxFX, Manjaro, OpenSUSE, PrimTux, Sabayon, SliTaz e Void Linux.

Cabe destacar que apesar do número de sistemas operacionais inicialmente pesquisados, nem todos tem aderência aos critérios inicialmente estabelecidos. Desse modo, considerando a tradução para o Português do Brasil, a disponibilidade de interface gráfica, a possibilidade de instalação de novos programas e que possuam uma

\footnotetext{
${ }^{4}$ Realizar a comparação do desempenho de determinado objeto executando programas de computador.
} 
comunidade ativa, as seguintes distribuições foram selecionadas: OpenSUSE, LinuxFX, Manjaro, Ubuntu Mate e Raspberry Pi OS.

Como último critério a ser avaliado, temos os testes de benchmark. Em computação, esse tipo de avaliação tem como objetivo mensurar um sistema quanto as questões de desempenho. Segundo Danaro et al. (2004), eles são:

1.Latência: tempo entre uma requisição e a completude de sua operação;

2.Vazão: o número de operações a ser concluídas em um período de tempo;

3.Escalabilidade: dependência entre o número de recursos que podem ser utilizados por um aplicativo distribuído.

Para realizar os testes supracitados, utilizou-se o Sysbench, um programa modular, multiplataforma e multi-threaded para avaliar os parâmetros de um sistema operacional. A opção por esse software deu-se em virtude da ferramenta ser amplamente utilizada para mensurar o desempenho de computadores de uso pessoal por usuários Linux. A Tabela 1 apresenta os módulos disponíveis para avaliação:

Tabela 1. Módulos disponíveis no Sysbench

\begin{tabular}{|l|l|}
\hline \multirow{2}{*}{ Drivers de banco de dados compilados } & \\
\hline \multirow{2}{*}{$\begin{array}{l}\text { mysql: benchmark de banco de dados } \\
\text { para o driver do MYSQL; }\end{array}$} & fileio: benchmark de entrada e saída de arquivos; \\
\cline { 2 - 2 } & cpu: benchmark de desempenho de CPU; \\
\cline { 2 - 2 } & memory: teste de velocidade de funções de memória RAM; \\
\hline \multirow{3}{*}{$\begin{array}{l}\text { pgsql: benchmark de banco de dados } \\
\text { para o driver do PostgreSQL. }\end{array}$} & threads: teste de desempenho do subsistema de threads; \\
\cline { 2 - 3 } & mutex: teste de desempenho Mutex; \\
\cline { 2 - 3 } & oltp: teste de desempenho de processamento em tempo real. \\
\hline
\end{tabular}

Fonte: Autores

Em virtude do escopo do projeto abarcar exclusivamente uma versão de baixo custo para computadores de uso pessoal, os testes foram focados na latência, utilizando os seguintes módulos: fileio, cpu, memory e threads.

Os sistemas operacionais Raspberry $P i \quad O S$ e Manjaro obtiveram um desempenho similar nos testes de benchmark. No entanto, o Raspberry Pi OS obteve um desenvolvimento superior em relação ao Manjaro em cinco (5) dos nove (9) testes realizados, representando um total de $55,56 \%$ dos casos. Sendo assim, o Raspberry Pi $O S$ foi selecionado para ser instalado no MINDER.

Dando continuidade a seleção dos softwares a serem instalados e configurados, temos o programa responsável pela captura das informações em tela e a respectiva conversão para o formato de áudio. A opção pelo Orca Screen Reader, deve-se prioritariamente por ser um leitor de telas gratuito e nativo em diversas distribuições Linux. Além da conversão em áudio do conteúdo disponível em tela, o programa também permite a ampliação de texto, tornando possível a interação de pessoas cegas e de baixa visão. Em relação as ferramentas de escritórios para manipular textos, planilhas e apresentações, optou-se por um programa que possua suporte as Tecnologias 
Assistivas, gratuito e que permita a instalação no Raspberry $P i O S$, a exemplo do LibreOffice. Por fim, a escolha do navegador seguiu as mesmas premissas do leitor de telas, ou seja, um programa gratuito e que seja disponibilizado nas diferentes versões do Linux.

\section{Metodologia}

Antes de adentrar no tópico que apresenta o método deste trabalho, torna-se necessário esclarecer que determinados encaminhamentos deram-se em função dos protocolos relacionados à pandemia do Sars-CoV-2, em particular o afastamento social. Diante desse contexto, tornou-se inviável a operacionalização de determinadas etapas existentes no percurso metodológico definido, a exemplo das fases que envolvem diretamente a presença de estudantes e pesquisadores em determinadas dinâmicas, como as oficinas temáticas e o processo de validação.

\subsection{Desvelando o processo de desenvolvimento do MINDER}

O Projeto MINDER fundamenta-se na Metodologia Colaborativa Aplicada, sendo definida pelo grupo de pesquisa em Geotecnologias, Educação e Contemporaneidade $\left(\right.$ GEOTEC $\left.^{5}\right)$ como: Colaborativa, por fomentar pesquisas de forma propositalmente coletiva, onde um conjunto de indivíduos possui a intencionalidade de interferir e sofrer interferência no/do coletivo; Aplicada, por gerar conhecimentos para aplicação e intervenção prática, dirigida à solução de problemas e/ou objetivos específicos. Neste trabalho, personificado pelo desenvolvimento da plataforma MINDER que intenciona minimizar e/ou suplantar as barreiras comunicacionais.

É justamente nesse contexto que as informações, proposições e resultados, emergem pela sinergia dos participantes envolvidos. As proposições dos estudantes, aqui entendidas como as necessidades relacionadas a superação das barreiras comunicacionais, fluem para os pesquisadores, sendo consideradas como insumos conceituais. Posteriormente, dar-se-á o retorno dos resultados, por parte dos pesquisadores, que se materializa através da aplicação de técnicas e métodos, relativos ao campo da computação objetivando o aperfeiçoamento da plataforma supracitada. Os resultados, personificados a título de exemplificação como atualizações e/ou correções de funcionalidades do MINDER são encaminhados aos estudantes para avaliação, que, por consequência, tecem as respectivas considerações (sugestões/críticas), retroalimentando a pesquisa.

É nesse universo que pesquisadores e estudantes encontram-se imersos, buscando presenciar e interpretar o maior número de situações manifestadas no objeto central, neste caso, a plataforma MINDER. As observações são interpretadas, tornandose fundantes na correção e/ou aperfeiçoamento da plataforma. Estes, por sua vez, retornam para novamente serem analisados (des/re/construir) e discutidos pelos estudantes.

Em continuidade ao percurso metodológico, tem-se a utilização de um conjunto de métodos adaptados: o ciclo PDCA (Planejamento, Execução, Checagem e Ação - do inglês Plan, Do, Check, Act) e o Processo de Desenvolvimento de Software Iterativo

\footnotetext{
${ }^{5} \mathrm{O}$ grupo discute e propõe ações relacionadas ao ensino, pesquisa e extensão nos diversos níveis de formação, a respeito do entendimento de Espaço mediado pelas TDIC.
} 
Incremental (PDSII). A adoção dos métodos supracitados, deu-se em virtude da aproximação da equipe técnica que compõe o projeto. Destaca-se ainda que, devido às particularidades dessa pesquisa, algumas diretrizes de ambos os métodos foram modificadas, substituídas ou excluídas, tornando possível alcançar os objetivos propostos.

A combinação dos métodos PDCA e PDSII foi idealizada para acompanhar o desenvolvimento do projeto, em particular pela flexibilidade e controle na melhoria contínua de processos e produtos. Dessa forma, utilizaremos o PDCA para controlar o projeto (processo e produto) na totalidade. Ou seja, na fase de Planejamento foram coletadas as necessidades dos estudantes com algum tipo de limitação visual, por meio das oficinas formativas e posteriormente encaminhada para etapa de Execução. Em relação a esta fase, traremos o PDSII (Concepção, Elaboração, Construção e Transição), especificamente, para o gerenciamento e operacionalização dos aspectos técnicos do hardware e do software (bits e bytes) da plataforma MINDER. A etapa de verificação se caracteriza como momento de checagem, onde existe o confronto das necessidades identificadas e o respectivo atendimento por meio dos requisitos técnicos implementados na plataforma referenciada anteriormente. Por fim, tem-se a fase da Ação, na qual as correções e/ou ajustes daquele ciclo serão efetuados. Desse modo, a junção desses dois métodos, está representada na Figura 2.

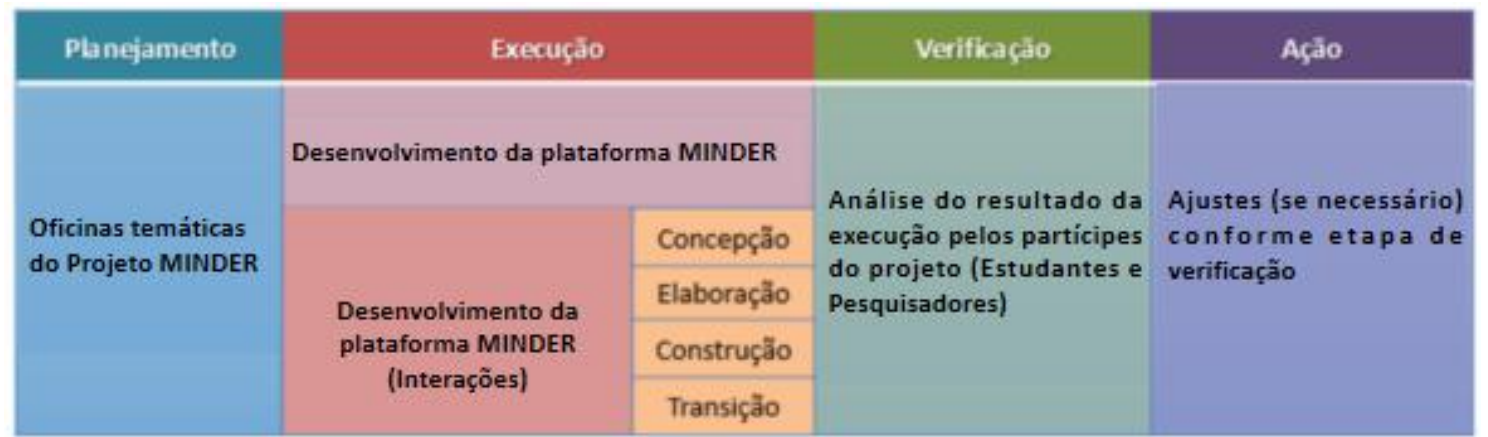

Figura 2 - Fluxo das informações relacionadas ao processo metodológico da plataforma MINDER.

Fonte: Autores

A dinâmica apresentada permite realimentar a pesquisa, de forma continua e cíclica, gerando novos requisitos, que por sua vez retornarão às oficinas temáticas, para (re)avaliações. Desse modo, objetiva-se o aprimoramento da plataforma, principalmente por basear-se nas necessidades do público-alvo, formado por alunos com deficiência visual, refletindo o envolvimento destes sujeitos no processo de desenvolvimento do MINDER, assim como nas descobertas e aprendizagens que o mesmo possibilita.

\section{Resultados preliminares}

Como dito anteriormente, este artigo é fruto de um projeto de pesquisa em fase de desenvolvimento. Sendo assim, temos como resultados preliminares a produção do hardware, um dispositivo composto por: um fone de ouvido, um teclado e uma central de processamento de dados (CPU). A implantação dos softwares que são necessários para operação do MINDER pelo deficiente visual, a saber: um (01) sistema operacional de código aberto, um (01) leitor de telas, um (01) conjunto de programas de escritório (planilha, texto e apresentação) e um navegador. 
Em virtude da pandemia, a plataforma MINDER não pode ser testada pelos usuários, o que adia os demais resultados esperados. Pretende-se obter os documentos mencionados na metodologia, bem como o estreitamento entre profissionais técnicos e pesquisadores do projeto, com a pretensão de gerar impactos na produção e difusão social, educacional e cultural e aproximação dos profissionais técnicos às necessidades da sociedade civil, no sentido de possibilitar a geração de produtos e/ou serviços que mobilizem as formas e as metodologias do campo da computação e educação.

A produção desse artefato foi posterior à etapa de Planejamento onde foram coletadas as necessidades dos estudantes com algum tipo de limitação visual, por meio das oficinas formativas, com base no método PDCA. Foram realizadas 2 oficinas formativas para coleta das informações sobre as necessidades dos estudantes e as possibilidades do dispositivo. Após a coleta, passou-se à etapa de Execução. Nesta fase, por meio do método PDSII (Concepção, Elaboração, Construção e Transição), foi construído o protótipo da plataforma MINDER (hardware e do software).

O terceiro momento pretende testar a plataforma MINDER em três (03) oficinas temáticas relacionadas a ambientação com o dispositivo e seus aplicativos. Nessa dinâmica, teremos a participação de cinco (05) estudantes com deficiência visual (cegos e/ou baixa visão). As oficinas objetivam a ambientação do uso da plataforma com vistas a apreensão, correção de eventuais falhas e melhorias, considerando que esta foi desenvolvida por pessoas videntes. Esta fase não pôde ser ainda executada devido a Pandemia da Covid-19, por tratar-se de necessários encontros presenciais, uma vez que pretende-se realizar a observação da interação dos participantes com a plataforma. Esta etapa corresponde à fase de checagem do ciclo PDCA (Planejamento, Execução, Checagem e Ação). A última etapa, a fase de Ação do método PDCA, diz respeito às intervenções necessárias na plataforma, após análise dos participantes.

Sendo assim, ao término das oficinas temáticas, espera-se como desdobramento a aproximação dos profissionais técnicos às necessidades da sociedade civil, no sentido de possibilitar a geração de produtos e/ou serviços que mobilizem as formas e as metodologias do campo da computação e educação. 


\section{Referências}

BOURNE, Rupert RA et al. Magnitude, temporal trends, and projections of the global prevalence of blindness and distance and near vision impairment: a systematic review and meta-analysis. The Lancet Global Health, v. 5, n. 9, p. e888-e897, 2017.

BRASIL. (1996) "Lei $\mathrm{n}^{\circ}$ 9.394, de 20 de dezembro de 1996", http://www.planalto.gov.br/ccivil_03/leis/19394.htm, Maio.

BRASIL. (2004) "Decreto $\mathrm{n}^{\mathrm{o}}$ 5.296, de 2 de dezembro de 2004", http://www.planalto.gov.br/ccivil_03/_ato2004-2006/2004/decreto/d5296.htm, Maio.

BRASIL. (2009) "Decreto $\mathrm{n}^{\mathrm{o}}$ 6.949, de 25 de agosto de 2009", http://www.planalto.gov.br/ccivil_03/_ato2007-2010/2009/decreto/d6949.htm, Maio.

BRASIL. (2015) "Lei $\mathrm{n}^{\mathrm{o}}$ 13.146, de 06 de julho de 2015", http://www.planalto.gov.br/ccivil_03/_ato2015-2018/2015/lei/113146.htm, Maio.

CONSELHO BRASILEIRO DE OFTALMOLOGIA. (2019) "As Condições de Saúde Ocular no Brasil "A 2019”, https://www.cbo.com.br/novo/publicacoes/condicoes_saude_ocular_brasil2019.pdf, Maio.

DOMINGUES, Celma dos Anjos. (2020) "Políticas públicas, difusão e mediação da tecnologia assistiva na perspectiva dos direitos humanos", Tese de Doutorado, Universidade Estadual de Campinas, Campinas.

FREIRE, Paulo. (2005) "Pedagogia do Oprimido. Rio de Janeiro", Paz e Terra.

GARCÍA, Jesus Carlos Delgado García; ITS BRASIL. (2017) "Livro Branco da Tecnologia Assistiva no Brasil", ITS BRASIL.

INSTITUTO BRASILEIRO DE GEOGRAFIA E ESTATÍSTICA. (2012) “Censo Demográfico 2010: Características gerais da população, religião e pessoas com deficiência",

https://biblioteca.ibge.gov.br/visualizacao/periodicos/94/cd_2010_religiao_deficienci a.pdf, Maio.

INSTITUTO BRASILEIRO DE GEOGRAFIA E ESTATÍSTICA. (2018) "Nota técnica 01/2018: Releitura dos dados de pessoas com deficência no Censo Demográfico 2010 à luz das recomendações do Grupo de Washington", https://ftp.ibge.gov.br/Censos/Censo_Demografico_2010/metodologia/notas_tecnica s/nota_tecnica_2018_01_censo2010.pdf, Maio.

INSTITUTO BRASILEIRO DE GEOGRAFIA E ESTATÍSTICA. (2020) "Síntese de Indicadores Sociais: Uma análise das condições de vida da população brasileira 2020”, https://biblioteca.ibge.gov.br/visualizacao/livros/liv101760.pdf, Maio

LAVORATO, Simone Uler. (2021) "Método Dialógico, Descritivo e Acessível DDA: uma estratégia pedagógica para adaptação de material didático para o ensino de ciências na perspectiva da escola inclusiva", Tese de Doutorado, Universidade Federal de Brasília, Brasília.

Gonzáles, E. (Org). Necessidades educacionais específicas: intervenção psicoeducacional. Porto Alegre: Artmed, 2007. 
Hogetop, L.; Santarosa, L.M.C, Tecnologias Adaptiva/Assistiva Informáticas na Educação Especial: viabilizando a acessibilidade ao potencial individual. Revista de Informática na Educação: Teoria, Prática. PGIE/UFRGS. V.5 N ${ }^{\circ} 2$ p.103-118 nov/2002.

Santarosa, Lucila M. C. Telemática y la inclusión virtual y social de personas con necesidades especiales: un espacio posible en la Internet - RIBIE 2000 - Chile. Disponível em: <http://www.c5.cl/ieinvestiga/actas/ribie2000>. Acesso em 08 mar. 2021.

OMS, Organização Mundial da Saúde. (2021) "Relatório mundial sobre a visão", https://apps.who.int/iris/bitstream/handle/10665/328717/9789241516570-por.pdf, Maio.

ROMÃO, E. S. e LIMA, M. F. M. "A educação no universo das imagens: tecnologia, cultura, linguagem", http://alb.com.br/arquivomorto/edicoes_anteriores/anais16/sem05pdf/sm05ss14_07.pdf, Maio.

WORLD HEALTH ORGANIZATION. (2018) "International Classification of Diseases 11th Revision", https://icd.who.int/en, Maio.

World Health Organization. (2018) "International Classification of Functioning, Disability and Health (ICF)", https://www.who.int/standards/classifications/international-classification-offunctioning-disability-and-health, Maio. 